\title{
Modal structure, directional and wavelength jumps of integrated semiconductor ring lasers: Experiment and theory
}

\author{
S. Fürst, ${ }^{1, a)}$ A. Pérez-Serrano, ${ }^{2}$ A. Scirè,${ }^{2}$ M. Sorel, ${ }^{1}$ and S. Balle ${ }^{3}$ \\ ${ }^{1}$ Department of Electronics and Electrical Engineering, University of Glasgow, Glasgow, Scotland G12 8LT, \\ United Kingdom \\ ${ }^{2}$ Instituto de Física Interdisciplinar y Sistemas Complejos (IFISC), CSIC-UIB, 07122 Palma de Mallorca, \\ Spain \\ ${ }^{3}$ Institut Mediterrani d'Estudis Avançats (IMEDEA), CSIC-UIB, 07190 Esporles, Spain
}

(Received 18 June 2008; accepted 6 November 2008; published online 24 December 2008)

We have experimentally and theoretically analyzed the modal properties of semiconductor ring lasers and the wavelength jumps that occur in connection with directional switching above the threshold. (C) 2008 American Institute of Physics. [DOI: 10.1063/1.3052601]

For many years, semiconductor ring lasers (SRLs) have attracted interest for their potential in the fabrication of microcavity lasers and densely integrated photonics circuits. It has recently been realized that the coexistence of two counterpropagating modes in the same gain medium leads to a large variety of operating regimes and dynamics that are profoundly different from those of Fabry-Perot lasers. ${ }^{1}$ One of the most interesting properties is the fast directional bistability that could be harnessed for all-optical memories and signal processing. ${ }^{2,3}$ Besides their switching characteristics, SRLs show several unexpected behaviors such as hysteresis in the lasing direction ${ }^{4,5}$ and atypical lasing mode selection rules. ${ }^{6}$ In particular, when current or temperature are changed, the lasing mode does not hop between consecutive cavity modes but exhibits sudden jumps between several cavity modes only when the lasing direction reverses. This characteristic strongly enhances the stability of the lasing wavelength against changes in the operating conditions.

In this letter we report on the measurement of the transfer function of SRL devices, which provides us with a map of the cavity resonances and the emission wavelength of the SRLs when biased above the threshold. The transfer function can be theoretically explained by considering the perturbation induced by the light-extraction sections, which induces symmetry breaking in the resonant cavity and modulation of the cavity losses. For the geometry considered, the cavity losses have a wavelength periodicity that corresponds to three ring cavity modes, which explains the measured hops in wavelength as the bias current of the laser is increased.

The device layout (see Fig. 1) consists of a ring cavity with a ring radius of $300 \mu \mathrm{m}$, coupled to a straight output waveguide by a point evanescent coupler. The waveguides are $2 \mu \mathrm{m}$ wide, and the gap between the ring and the output waveguides is $750 \mathrm{~nm}$, providing a theoretical coupling ratio of $12 \%$. To minimize the backreflections, the output waveguides are $10^{\circ}$ tilted to the cleaved facets.

The wafer used to fabricate the devices is a multiple quantum well AlGaInAs/InP structure, grown by metalorganic chemical vapor deposition (CVD). The waveguides were defined by electron beam lithography and transferred to a plasma enhanced $\mathrm{CVD} \mathrm{SiO}$ layer using $\mathrm{CHF}_{3}$ reactive ion etching (RIE). A shallow etched ridge-waveguide was then

${ }^{a)}$ Electronic mail: furst@elec.gla.ac.uk. defined by RIE using a chemistry of the $\mathrm{CH}_{4} / \mathrm{H}_{2} / \mathrm{O}_{2}$ process, which is selective to the $\mathrm{Al}$ containing core layer and thus ensures a very good control over the etching depth and the power coupling ratio. The etching depth provides an effective refractive index difference of $\Delta n=0.064$, which makes the bending losses negligible down to ring radii of $140 \mu \mathrm{m}$. ${ }^{7}$ The subsequent deposition of $\mathrm{a} \mathrm{SiO}_{2}$ layer was followed by a contact window definition for current injection. Finally, metal contacts were deposited on both the epitaxial and substrate sides of the wafer section.

For analyzing the cavity resonances of the SRL, we inject through port 1 a monochromatic field from a tunable laser, and we measure the photocurrent generated in ports 3 and 4, which are reverse biased (see Fig. 1). During these measurements, the ring is biased close to transparency to minimize the losses. The power collected at port 3 as the input wavelength is scanned (positive peaks in Fig. 2) displays narrow and well defined peaks at wavelengths equispaced by $0.4 \mathrm{~nm}$. The peak heights show the expected profile defined by the wavelength-dependent gain spectrum in the structure and an additional modulation that occurs every three longitudinal modes.

Measurements over different devices show that the longitudinal modes possess a doublet structure, with the splitting between the two subpeaks varying from 1 to $4 \mathrm{GHz}$. In addition, for a particular device, the splitting between the subpeaks usually displays a modulation that corresponds

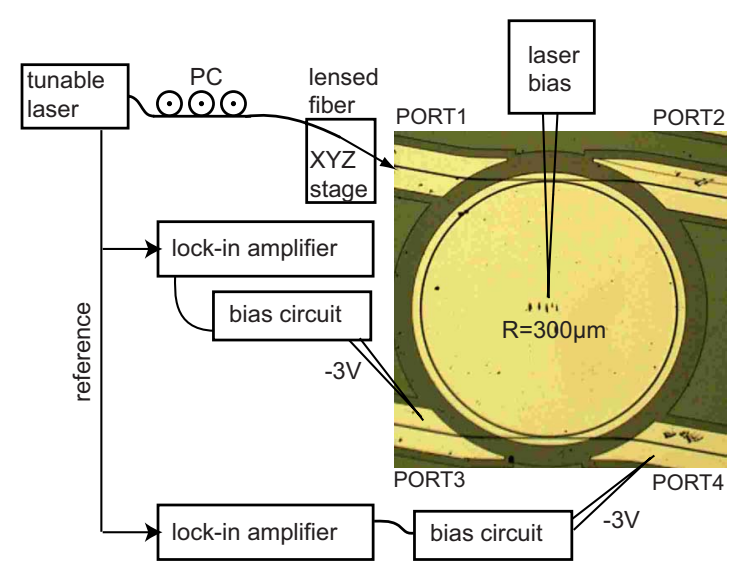

FIG. 1. (Color online) Optical micrograph of a $300 \mu \mathrm{m}$ radius ring laser with the corresponding measurement setup. 


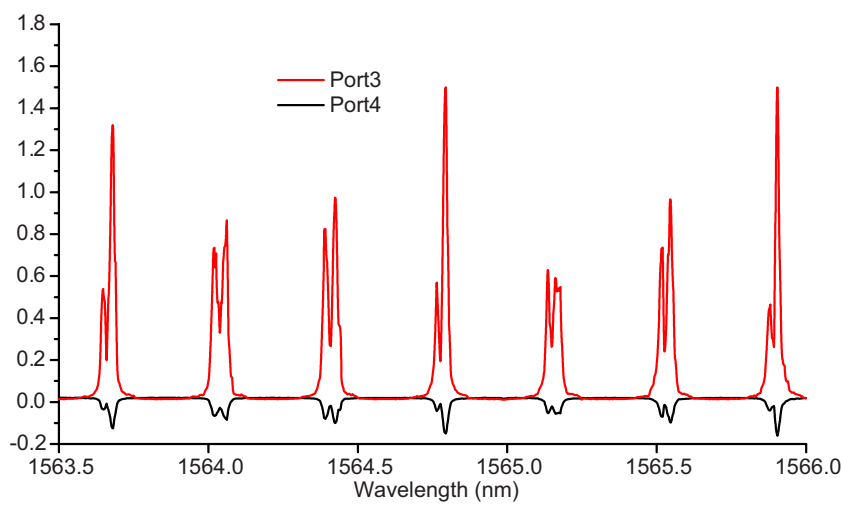

FIG. 2. (Color online) Detected power (arbitrary units) at ports 3 and 4 .

with the additional modulation in the longitudinal mode spectrum described above. Figure 3 shows the measured doublet splitting for another device, which displays a modulation that also corresponds to a periodicity of roughly three mode spacings.

The power collected at port 4 (negative peaks in Fig. 2) presents a similar structure with the same periodicity, but instead of displaying peaks above a spontaneous-emission noise background, it shows dips on such a background. It is worth remarking that the depth of the dips strongly depends on the bias current in the SRL cavity (see Fig. 4): the dips cannot be seen for bias currents below $30.5 \mathrm{~mA}$, but they are visible above.

When the laser is biased above the threshold, the main lasing direction does not remain stable for all current values. The $L-I$ curve for the device in Fig. 3 shows periodic switching between clockwise $(\mathrm{CW})$ and counterclockwise (CCW) emissions for increasing current, a generic behavior in this type of devices. ${ }^{4}$ Additionally, the dominant lasing wavelength remains constant except for a small thermal drift between switches, but it suddenly jumps by three cavity modes when the lasing direction reverses, as shown in Fig. 5.

The experimental results below the threshold can be explained by computing the transfer matrix of the complete SRL structure. ${ }^{8}$ We assume that the two couplers are identical, lossless, and with a residual reflectivity due to their pointlike character. Small reflectivity from output facets 1 and 2 is included, but not from facet 3 or 4 since the corresponding output waveguides are reverse biased. From this analysis, the roundtrip condition for the SRL modes in a

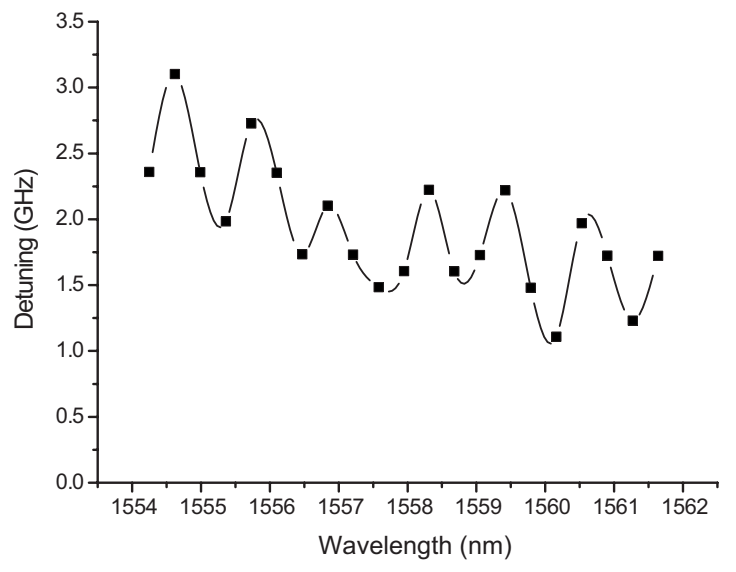

FIG. 3. Measured splitting between doublets.

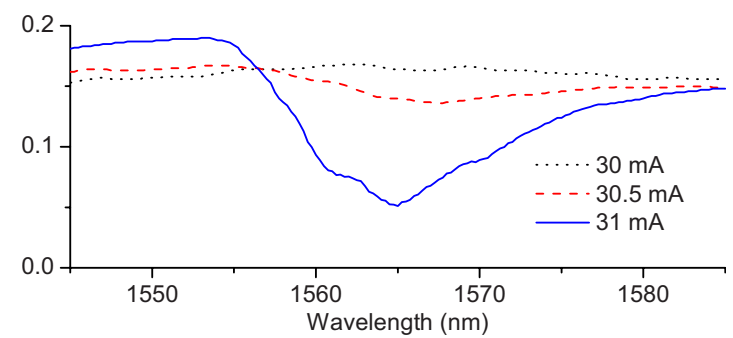

FIG. 4. (Color online) Current dependence of the dips at port 4 .

resonator with equal arms of length $L / 2$ can be formulated as

$$
e^{2 i q L}-a e^{i q L}+b=0,
$$

where $q$ is the complex propagation constant. In Eq. (1), $b$ $=\left(r_{u} r_{u}^{\prime}-t_{u} t_{u}^{\prime}\right)^{-1}\left(r_{d} r_{d}^{\prime}-t_{d} t_{d}^{\prime}\right)^{-1}$ and $a=\left(r_{u} r_{d}+r_{u}^{\prime} r_{d}^{\prime}+t_{u}^{\prime} t_{d}+t_{u} t_{d}^{\prime}\right) b$; $t_{u(l)}$ and $r_{u(l)}$ denote the frequency-dependent transmittivity and reflectivity of the upper (lower) coupler for $\mathrm{CW}$ waves, and primed symbols denote the same magnitudes for $\mathrm{CCW}$ waves. Defining $Q_{ \pm}=a / 2 \pm\left[(a / 2)^{2}-b\right]^{1 / 2}$, the modes are given by

$$
q_{m}^{ \pm} L=2 \pi m-i \ln Q_{ \pm} .
$$

The light-extraction section breaks the circular symmetry of the SRL, ${ }^{9}$ destroying the pure $\mathrm{CW}$ and $\mathrm{CCW}$ states at $q_{m}$ $=2 \pi m$. Two branches of solutions emerge due to the term $-i \ln Q_{ \pm}$, which correspond to the experimentally observed doublets. Their splitting, normalized to the free-spectral range of the SRL, is thus given by

$$
\Delta=\frac{1}{2 \pi}\left\{\operatorname{Im}\left[\ln \left(\frac{Q_{-}}{Q_{+}}\right)\right]-\alpha \operatorname{Re}\left[\ln \left(\frac{Q_{-}}{Q_{+}}\right)\right]\right\},
$$

$\alpha$ being the linewidth enhancement factor.

The theoretical results for the power at port 3 are in good agreement with the results shown in Fig. 2, provided that a small amount of gain in the SRL is included (see Fig. 6). In these calculations, the section lengths have been taken from the device layout, and facet reflectivities have been adjusted to match the experimental results.

The results for the transfer matrix to port 4 show similar trends, and the transfer matrix analysis does not lead to dips on a spontaneous-emission noise background. The reason is that the transfer matrix analysis does not include spontaneous-emission noise in the SRL cavity. Indeed, due to the (slight) gain in the SRL, the power collected at ports 3

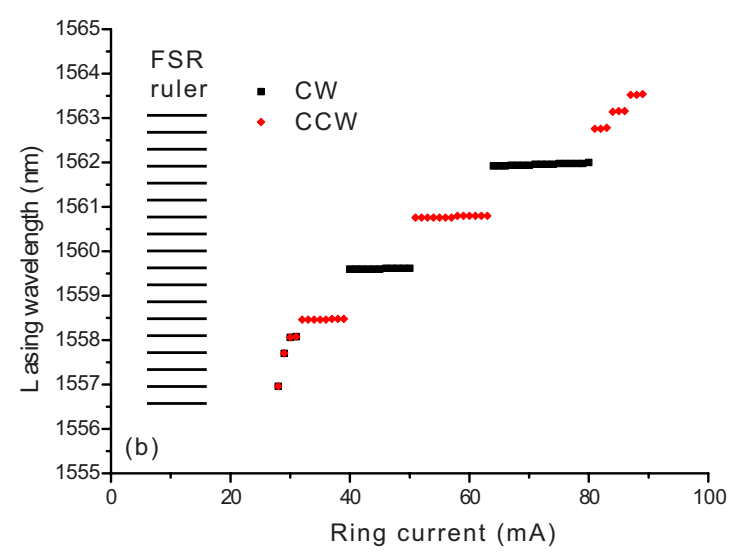

FIG. 5. (Color online) Lasing wavelength as a function of the SRL current. 


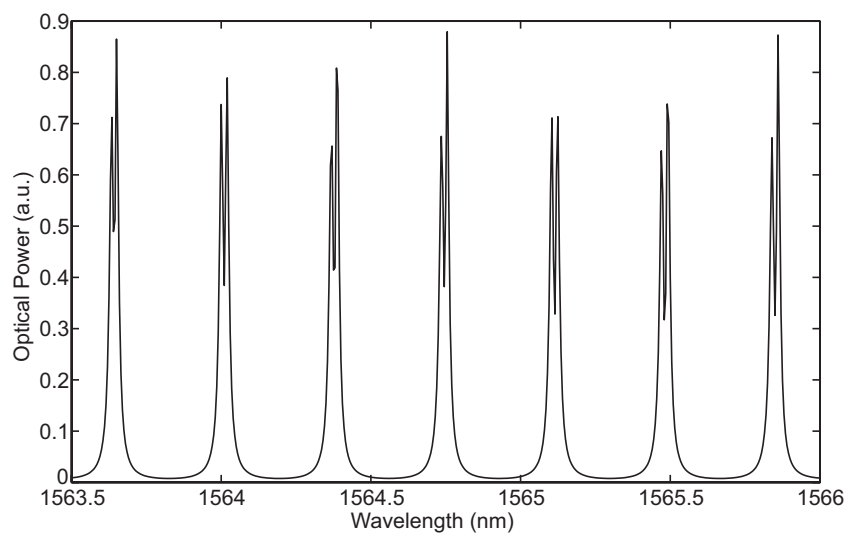

FIG. 6. Theoretical calculation of the power collected at port 3 .

and 4 in the absence of external light is the power due to spontaneous emission in the SRL, including amplification and attenuation in the path. In the absence of any reflecting element, light injected into the SRL through port 1 would reach port 3 only after being amplified or attenuated along the path, and no injected light would reach port 4; however, the power at port 4 would be reduced because of amplified spontaneous-emission (ASE) suppression under light injection, thus leading to dips onto the ASE background. It is worth remarking that this effect provides us with a precise way to measure the spectral dependence of the transparency current.

In the same way, we can compute the theoretical detuning for the device used in Figs. 3 and 5 (see Fig. 7). The modulation of the detuning at three mode spacings is apparent, arising from the residual reflectivity at the bends of the output waveguides. In addition, it presents a further slow modulation that arises from the finite effective length of the output couplers and their residual reflectivities.

Finally, the wavelength jumps of several modes above the threshold can be partially explained with the same analysis, although a full explanation of the above-threshold phenomenology requires considering nonlinear effects arising from, e.g., four-wave mixing ${ }^{10}$ and spatial and spectral hole

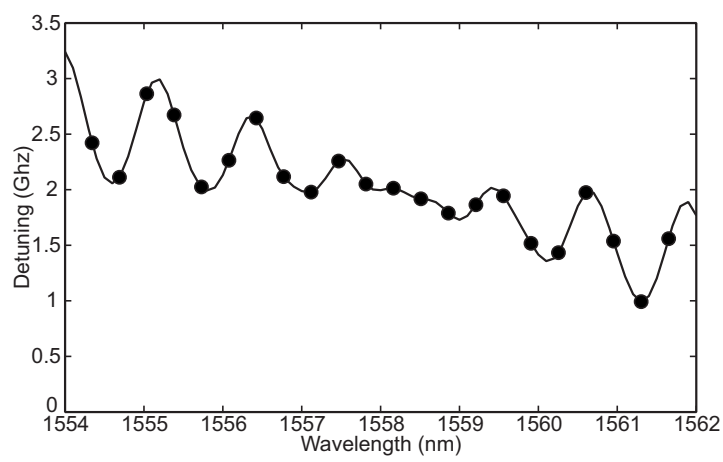

FIG. 7. Theoretical detuning between doublets. burning. ${ }^{11}$ From Eqs. (1) and (3) we see that for $\alpha \gtrsim 2-3$, the maximum frequency splitting of the doublets almost coincides with their maximum threshold difference, whose modulation for each branch is out of phase. Hence, when the gain spectrum redshifts due to Joule heating, the system will jump from the minimum on one branch to the following minimum on the other branch. For circular SRL, where $L$ $\geq 2 R$, this means a jump of $m=\operatorname{int}\left[3 \tau_{R} / \tau_{\mathrm{FP}}\right]$ modes of the SRL, where $\tau_{R(\mathrm{FP})}$ is the roundtrip time in the SRL (FabryPerot) cavity. Thus, for the device considered here, the modal jumps correspond to $m=3$.

In summary, we have experimentally studied the modal structure of SRLs by measuring the transfer properties of the device below the threshold and the wavelength jumps that occur in connection with directional switching above the threshold. A transfer matrix analysis explains the measurements when ASE in the SRL cavity is accounted for. The residual reflectivities in the light-extraction sections determine the frequency splitting and threshold difference between the two branches of solutions. This effect, together with the redshift of the material gain, explains the amount of wavelength jumps displayed by the SRL above the threshold.

We acknowledge financial support from the IOLOS (034743) project funded by the European Union's Sixth Framework Program (EU FP6-2005-IST-5), the PhoDeCC (TEC2006-10009/MIC) project funded by the Spanish Ministerio de Educación y Ciencia (MIC), the QULMI (PROGECIB-5A) project funded by the Spanish Govern Balear, and the TEC2006-13887-C05-03 project funded by the Spanish Ministerio de Educación y Ciencia.

${ }^{1}$ M. Sorel, G. Giuliani, A. Scirè, R. Miglierina, S. Donati, and P. J. R. Laybourn, IEEE J. Quantum Electron. 39, 1187 (2003).

${ }^{2}$ V. R. Almeida, C. A. Barrios, R. R. Panepucco, M. Lipson, M. A. Foster, D. G. Ouzounov, and A. L. Gaeta, Opt. Lett. 29, 2867 (2004).

${ }^{3}$ M. T. Hill, H. J. S. Dorren, T. de Vrie, X. J. M. Leijtens, J. H. den Besten, B. Smalbrugge, Y. S. Oei, H. Binsma, G. D. Khoe, and M. K. Smit, Nature (London) 432, 206 (2004).

${ }^{4}$ M. Sorel, P. J. R. Laybourn, G. Giuliani, and S. Donati, Appl. Phys. Lett. 80, 3051 (2002).

${ }^{5}$ M. F. Booth, A. Schremer, and J. M. Ballantyne, Appl. Phys. Lett. 76, 1095 (2000)

${ }^{6}$ C. J. Born, M. Hill, S. Yu, and M. Sorel, Quantum Electronics and Laser Science Conference 2005 (QELS '05), 22-27 May 2005 (unpublished), Vol. 2, p. 1035.

${ }^{7}$ S. Fürst, M. Sorel, A. Scirè, G. Giuliani, and S. Yu, Photonics Europe (SPIE, Strasbourg, France, 2006).

${ }^{8}$ A. E. Siegman, Lasers (University Science Books, Mill Valley, CA, 1986), pp. 398-426.

${ }^{9}$ E. J. D'Angelo, E. Izaguirre, G. B. Mindlin, G. Huyet, L. Gil, and J. R. Tredicce, Phys. Rev. Lett. 68, 3702 (1992).

${ }^{10}$ C. Born, G. Yuan, Z. Wang, M. Sorel and S. Yu, "Lasing mode hysteresis characteristics in semiconductor ring lasers," IEEE J. Quantum Electron. (in press).

${ }^{11}$ A. Uskov, J. Mork, and J. Mark, IEEE J. Quantum Electron. 30, 1769 (1994). 